Jaurnal of Environmental Science, Computer Science and Engineering \& Technology

An International Peer Review E-3 Journal of Sciences and Technology

\author{
Available online at www.jecet.org
}

Section B: Computer Science

Research Article

\title{
Window method for digital speech files cryptography
}

\author{
Dr. Hatim Zaini ${ }^{1}$ and Prof. Ziad A. Alqadi ${ }^{2}$ \\ ${ }^{1}$ Taif University, KSA, Ta'if Saudi Arabia \\ ${ }^{2}$ Albalqa applied university, Jordan
}

Received: 28September 2021; Revised: 15 October 2021; Accepted: 22 October 2021

\begin{abstract}
Speech signals some time require encryption to secure them from the hacker, in this article research a simple, and easy to implement method of digital speech file cryptography will be introduced. The suggested window method will be implemented to prove that the method satisfies the requirements of cryptography by giving good values of the quality parameters after the encryption and decryption. The introduced method will be compared with DES method to show how the proposed method will increase the efficiency and cryptography throughput.
\end{abstract}

Key words: Speech, PK, window, cryptography, throughput, PSNR.

\section{INTRODUCTION}

Speech signal ${ }^{[1,2]}$ is a set of samples, each of them represents the amplitude value for the corresponding analogue value sampled and quantized at a period of time, this signal can be represented by a one column matrix (mono speech) or two column matrix (stereo speech), these matrices can be easily maintained to apply a necessary operation using the speech signal ${ }^{[3-5]}$.

The speech file is used in many vital applications and is circulated through various means of communication ${ }^{[6-8]}$, and this file may be confidential, which requires protection ${ }^{[9]}$ from tampering or intruders or from any third party that is not authorized to hear and understand the speech ${ }^{[10]}$. One of the important methods used to protect voice and voice messages is the speech cryptography method, which includes the implementation of two operations: speech encryption and speech decryption ${ }^{[11.12]}$. 
Encryption and decryption operations can be maintained using private key (PK) and by performing some arithmetic and logic operation as show in figure 1.

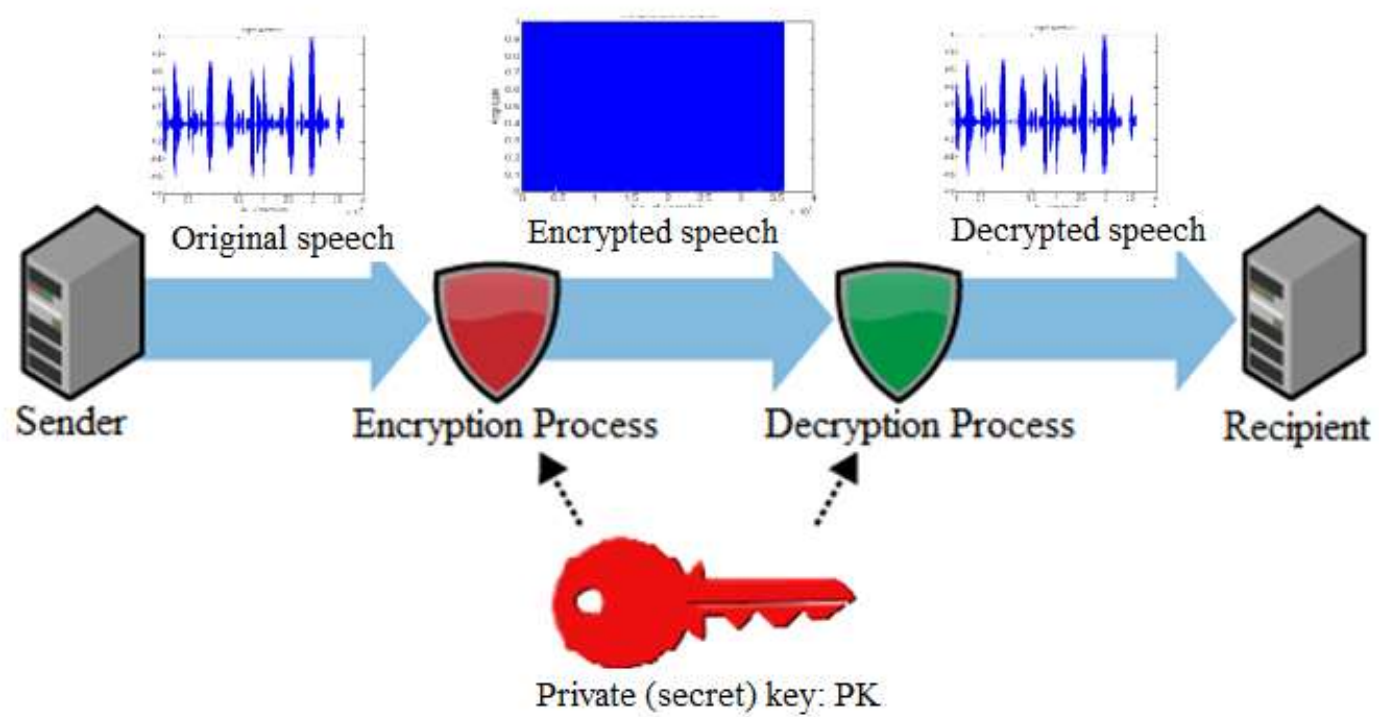

Figure 1: Speech cryptography

In order to increase the level of protection of the speech file, the private key must be complex in such a way that it is impossible to hack and be known only by the sender and receiver. Accordingly, the encryption process must destroy the speech file and distort it so that it cannot be understood by any unauthorized third party, as for the decryption process, it must work to return the original file without changing or without losing any information ${ }^{[13-15]}$.

The efficiency of the encryption and decoding method is measured by the time required to execute each of them, but the quality of the cryptography can be measured by the quality parameters PSNR (peak signal to noise ratio) and MSE (mean square error), and here the method of cryptography must satisfy the following ${ }^{[16-22]}$ :

1. MSE between the original speech and the encrypted file must be very big (full destruction), or the PSNR between them must be very low.

2. MSE between the original speech and the decrypted file must be very low or closed to zero (full recovery), or the PSNR between them must be very high or closed to infinite.

MSE and PSNR can be calculated using equations 1 and 2:

$$
\begin{gathered}
M S E=\frac{1}{m n} \sum_{0}^{m-1} \sum_{0}^{n-1}\|f(i, j)-g(i, j)\|^{2} \\
P S N R=20 \log _{10}\left(\frac{M A X_{f}}{\sqrt{M S E}}\right)
\end{gathered}
$$

Where: $f$ and $g$ are the two speech files to be compared for quality. 


\section{RELATED WORKS}

Many methods ${ }^{[23]}$ used for data cryptography are based on data decryption standard (DES). DES uses a 56-bit PK and the data to be divided into blocks of 64 bit each as shown in figure 2. DES requires: Round function, key scheduling, and additional processing - Initial and final permutation in both encryption and decryption processes.

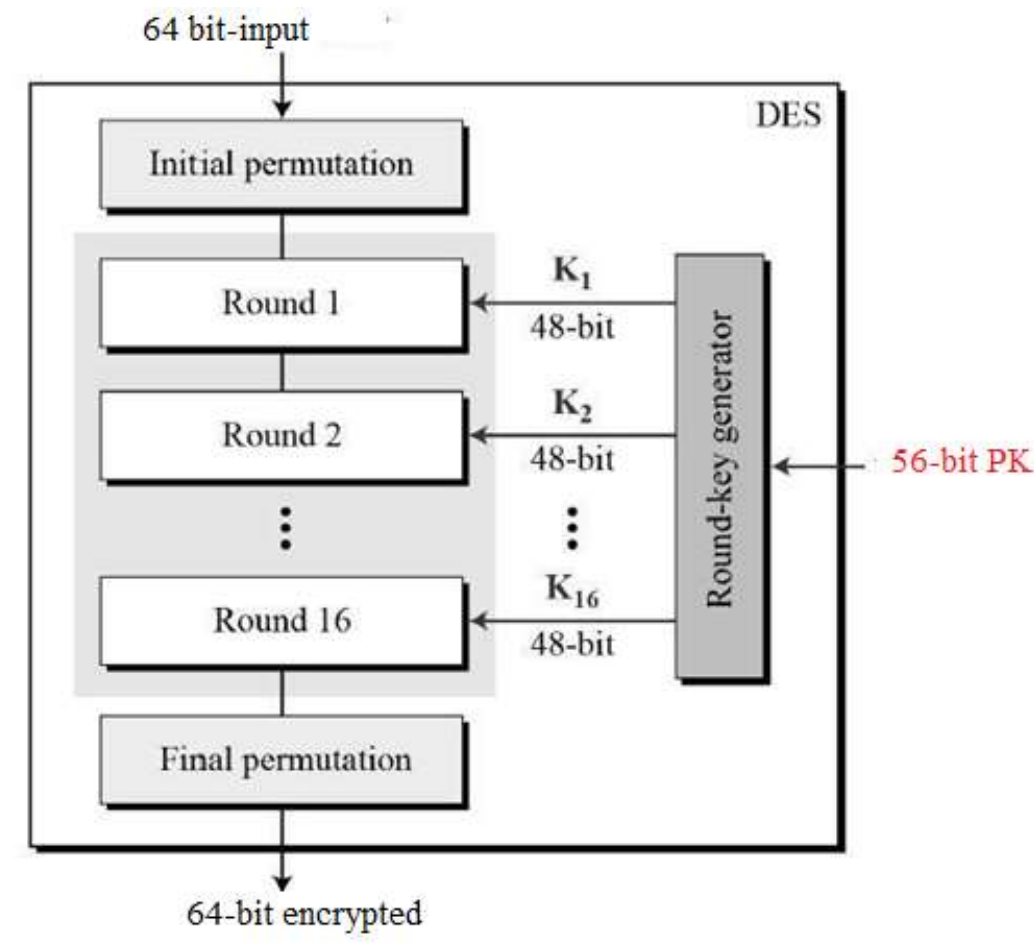

Figure 2: DES encryption

DES based methods ${ }^{[24]}$ are not enough secure because the private key can be hacked, also it requires some arithmetic and logical operation which required hard effort to implement them using double fractional data leading to increase the encryption-decryption times.

\section{MATERIALS AND METHODS}

The proposed method: The proposed method takes the speech signal and divides into equal size windows, a PK key will be used, this key will be generated and saved by the sender and receiver, and PK size must equal window size. Here window size selection is flexible and it can be changed when needed, the elements of the PK array must be within the range -2 to 2 .

The encryption phase can be implemented applying the following steps (figure 3):

- $\quad$ Get the speech signal file (matrix).

- $\quad$ Select the window size.

- $\quad$ Get the PK.

- $\quad$ For each window in the speech signal file add PK to it to get the encrypted window and at the end the encrypted speech file. 


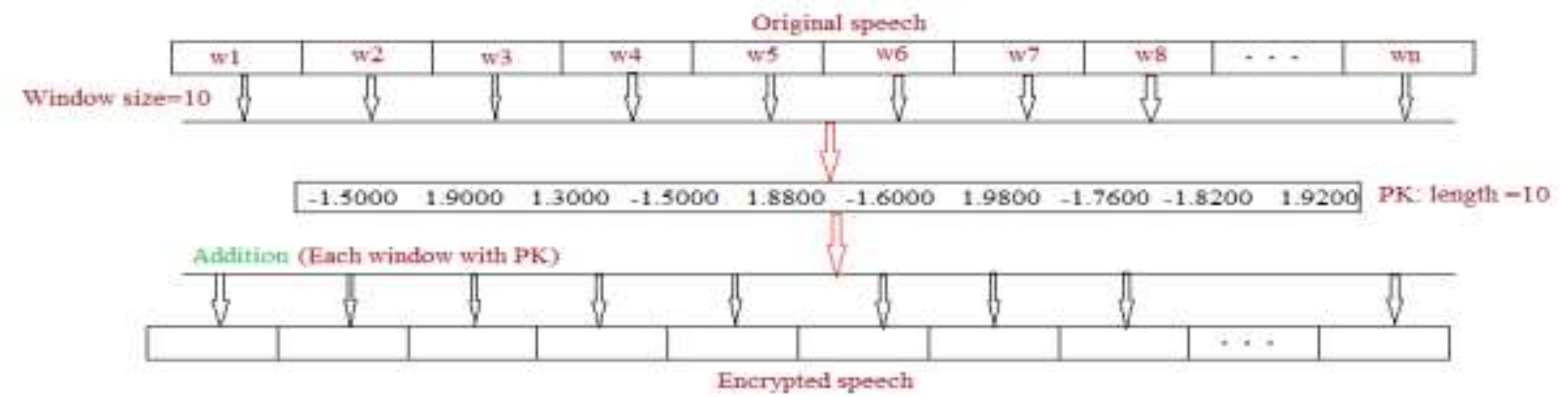

Figure 3: Encryption phase

The decryption phase can be implemented applying the following steps ( figure 4):

- $\quad$ Get the encrypted speech signal file.

- $\quad$ Select the window size.

- Get the PK.

- For each window in the speech signal file subtract PK from it to it to get the decrypted window and at the end the decrypted speech file.

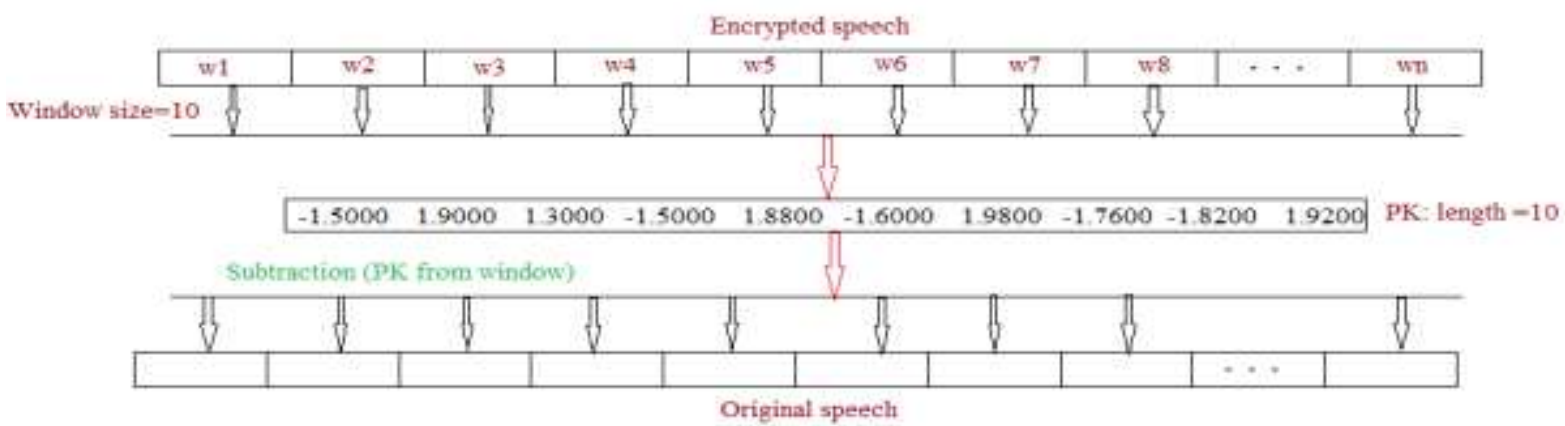

Figure 4: Decryption phase

\section{RESULT AND DISCUSSION}

Implementation and experimental results: The proposed method was implemented using various speech files, figure 5 shows an output sample of one of the implementations. To perform comparisons, DES method was programmed using matlab, this program was implemented using various speech file, and table 1 shows the obtained experimental results

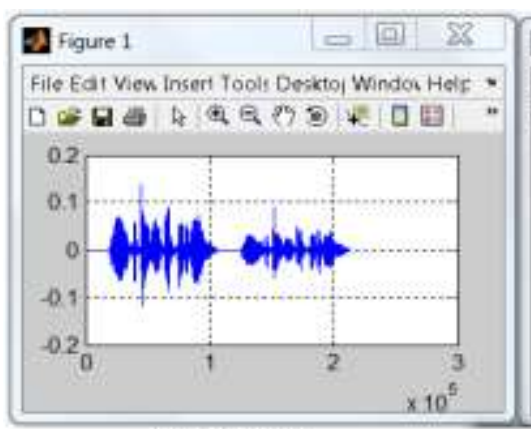

Original speech

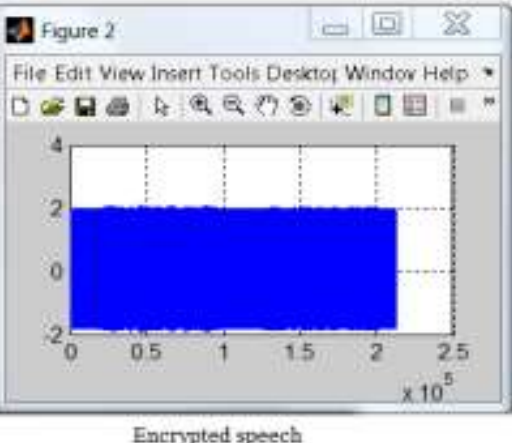

Enerypted speech

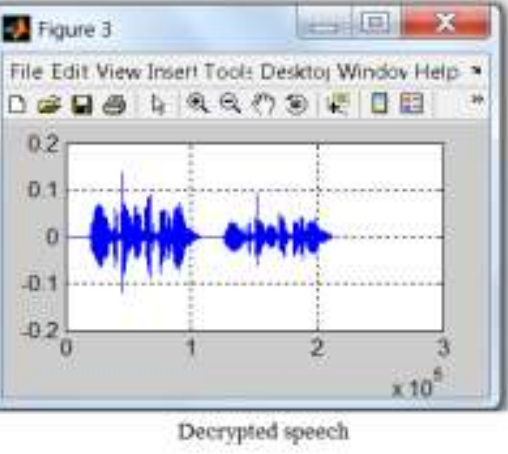

Decrypted speech

Figure 5: Sample outputs 
Table 1: DES results

\begin{tabular}{|c|c|c|c|c|c|}
\hline Speech signal & Size(samples) & $\begin{array}{l}\text { PSNR(original } \\
\text { and encrypted) }\end{array}$ & $\begin{array}{l}\text { PSNR(original } \\
\text { and decrypted) }\end{array}$ & $\begin{array}{c}\text { Encryption } \\
\text { time(seconds) }\end{array}$ & $\begin{array}{c}\text { Decryption } \\
\text { time(seconds) }\end{array}$ \\
\hline 1 & 321536 & 5.7842 & Infinite & 78.0480 & 77.2400 \\
\hline 2 & 200704 & 5.7708 & Infinite & 46.7060 & 47.8480 \\
\hline 3 & 227328 & 5.7867 & Infinite & 53.4560 & 53.1560 \\
\hline 4 & 430080 & 5.7885 & Infinite & 107.5440 & 107.8880 \\
\hline 5 & 172032 & 5.7536 & Infinite & 40.0840 & 39.9020 \\
\hline 6 & 133120 & 6.3252 & Infinite & 30.8140 & 30.3300 \\
\hline 7 & 212992 & 4.7012 & Infinite & 49.8000 & 50.5940 \\
\hline 8 & 272384 & 6.3332 & Infinite & 65.5740 & 65.5960 \\
\hline 9 & 82880 & 6.3252 & Infinite & 19.4410 & 19.2120 \\
\hline 10 & 64448 & 6.3284 & Infinite & 14.750000 & 14.980000 \\
\hline 11 & 122816 & 8.6305 & Infinite & 29.104000 & 29.600000 \\
\hline 12 & 138176 & 9.8083 & Infinite & 33.224000 & 33.167000 \\
\hline Average & 198208 & & & 47.3788 & 47.4594 \\
\hline \multicolumn{4}{|c|}{ Throughput(size/time) sample per second } & 4183.5 & 4176.4 \\
\hline
\end{tabular}

From table 2 we can see that the proposed method keeps the values of the quality parameters good enough, the encryption-decryption times for speeches with big sizes remain high, but for smaller speeches the times were decreased significantly (see value with red and green colors in table 2), thus the method throughput was increased. Now we use a window size of 20 samples, and a private key of 20 elements, table 3 shows the implementation results using these values.

Table 2: Proposed method results $(\mathrm{W}=10)$

\begin{tabular}{|c|c|c|c|c|c|}
\hline Speech signal & Size(samples) & $\begin{array}{l}\text { PSNR(original } \\
\text { and encrypted) }\end{array}$ & $\begin{array}{l}\text { PSNR(original } \\
\text { and decrypted) }\end{array}$ & $\begin{array}{l}\text { Encryption } \\
\text { time(seconds) }\end{array}$ & $\begin{array}{l}\text { Decryption } \\
\text { time(seconds) }\end{array}$ \\
\hline 1 & 321536 & 5.2123 & 784.4711 & 80.438000 & 81.292000 \\
\hline 2 & 200704 & 4.9732 & 783.9778 & 29.926000 & 30.548000 \\
\hline 3 & 227328 & 4.9297 & 779.1673 & 38.300000 & 39.136000 \\
\hline 4 & 430080 & 5.1497 & 785.3343 & 139.801000 & 140.462000 \\
\hline 5 & 172032 & 3.5339 & Infinite & 22.098000 & 22.335000 \\
\hline 6 & 133120 & 3.3160 & Infinite & 12.994000 & 13.040000 \\
\hline 7 & 212992 & 3.8220 & Infinite & 34.422000 & 35.552000 \\
\hline 8 & 272384 & 4.1407 & Infinite & 57.276000 & 57.886000 \\
\hline 9 & 82880 & 3.7969 & Infinite & 5.053000 & 4.748000 \\
\hline 10 & 64448 & 3.6915 & Infinite & 2.642000 & 2.688000 \\
\hline 11 & 122816 & 3.5324 & Infinite & 10.907000 & 10.998000 \\
\hline 12 & 138176 & 3.4711 & Infinite & 14.111000 & 14.294000 \\
\hline Average & 198208 & & & 37.3307 & 37.7482 \\
\hline \multicolumn{4}{|c|}{ Throughput(samples per second) } & 5309.5 & 5250.8 \\
\hline
\end{tabular}

From table 3 we can see that both the encryption and decryption times decreased and this will lead to increase the method throughput making the method more efficient.

Another smaller in size speeches were selected and implemented using DES and the proposed method, table 4 shows the obtained results for both methods, and from this table we can see that the proposed method is more efficient comparing with DES method and the proposed method significantly decrease the encryption and decryption times, and thus it increases the encryptiondecryption processes throughputs, this is shown in figures 6 and 7 
Table 3: Proposed method results $(\mathrm{W}=20)$

\begin{tabular}{|c|c|c|c|}
\hline Speech signal & Size(samples) & Encryption time(seconds) & Decryption time(seconds) \\
\hline 1 & 321536 & 39.505000 & 39.665000 \\
\hline 2 & 200704 & 14.976000 & 15.364000 \\
\hline 3 & 227328 & 19.373000 & 19.893000 \\
\hline 4 & 430080 & 70.371000 & 70.134000 \\
\hline 5 & 172032 & 10.963000 & 11.116000 \\
\hline 6 & 133120 & 6.336000 & 6.460000 \\
\hline 7 & 212992 & 17.265000 & 17.340000 \\
\hline 8 & 272384 & 28.067000 & 28.192000 \\
\hline 9 & 82880 & 2.492000 & 2.345000 \\
\hline 10 & 64448 & 1.349000 & 1.375000 \\
\hline 11 & 122816 & 5.552000 & 5.491000 \\
\hline 12 & 138176 & 6.975000 & 7.094000 \\
\hline
\end{tabular}

Table 4: DES and proposed method efficiency factors

\begin{tabular}{|c|c|c|c|c|c|}
\hline & & \multicolumn{2}{|c|}{ DES time(seconds) } & \multicolumn{2}{c|}{ Proposed time(Seconds) } \\
\hline Speech number & Size(samples) & Encryption & Decryption & Encryption & Decryption \\
\hline 13 & 98015 & 22.542000 & 22.506000 & 6.935000 & 6.868000 \\
\hline 14 & 94819 & 21.821000 & 21.823000 & 6.440000 & 6.404000 \\
\hline 15 & 84691 & 19.525000 & 19.649000 & 5.154000 & 5.011000 \\
\hline 16 & 157413 & 37.753000 & 37.597000 & 18.392000 & 18.194000 \\
\hline 17 & 67083 & 15.149000 & 15.440000 & 2.956000 & 3.027000 \\
\hline 18 & 127146 & 30.219000 & 29.788000 & 11.844000 & 12.046000 \\
\hline 19 & 110344 & 25.786000 & 25.811000 & 8.846000 & 8.824000 \\
\hline 20 & 122692 & 28.786000 & 28.736000 & 10.943000 & 11.159000 \\
\hline 21 & 129790 & 30.522000 & 30.514000 & 12.258000 & 12.411000 \\
\hline 22 & 161565 & 38.785000 & 39.056000 & 19.195000 & 19.567000 \\
\hline & & & & & \\
\hline
\end{tabular}

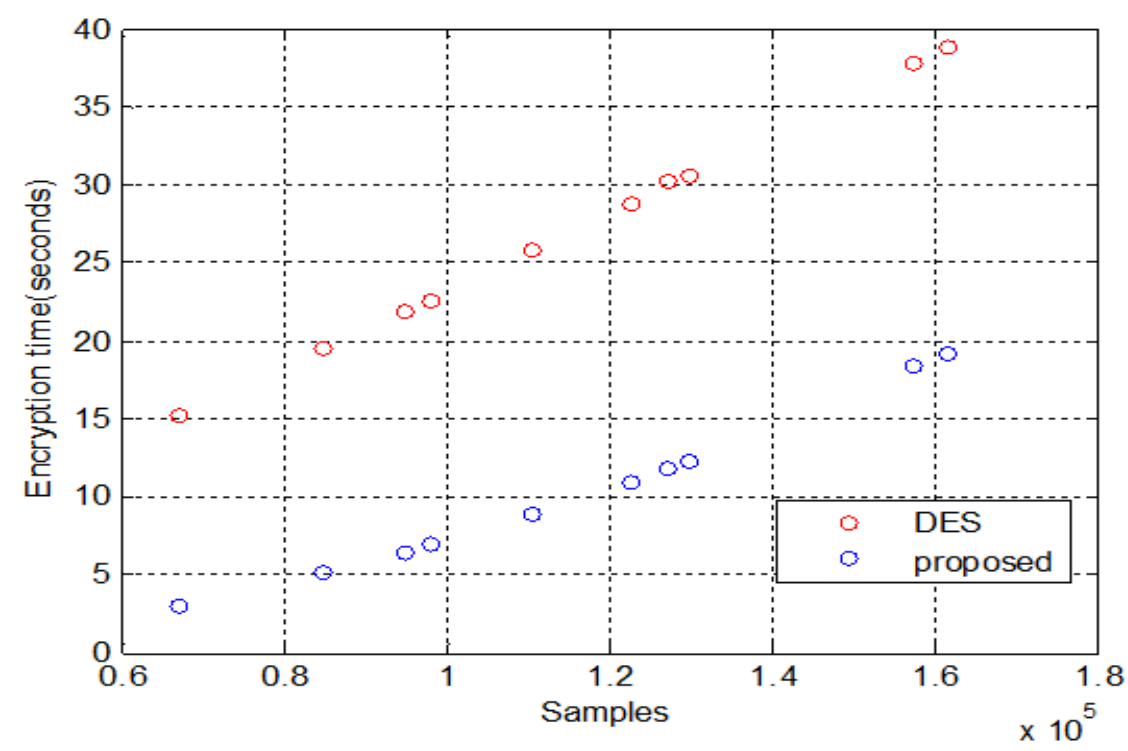

Figure 6: Encryption times comparisons 


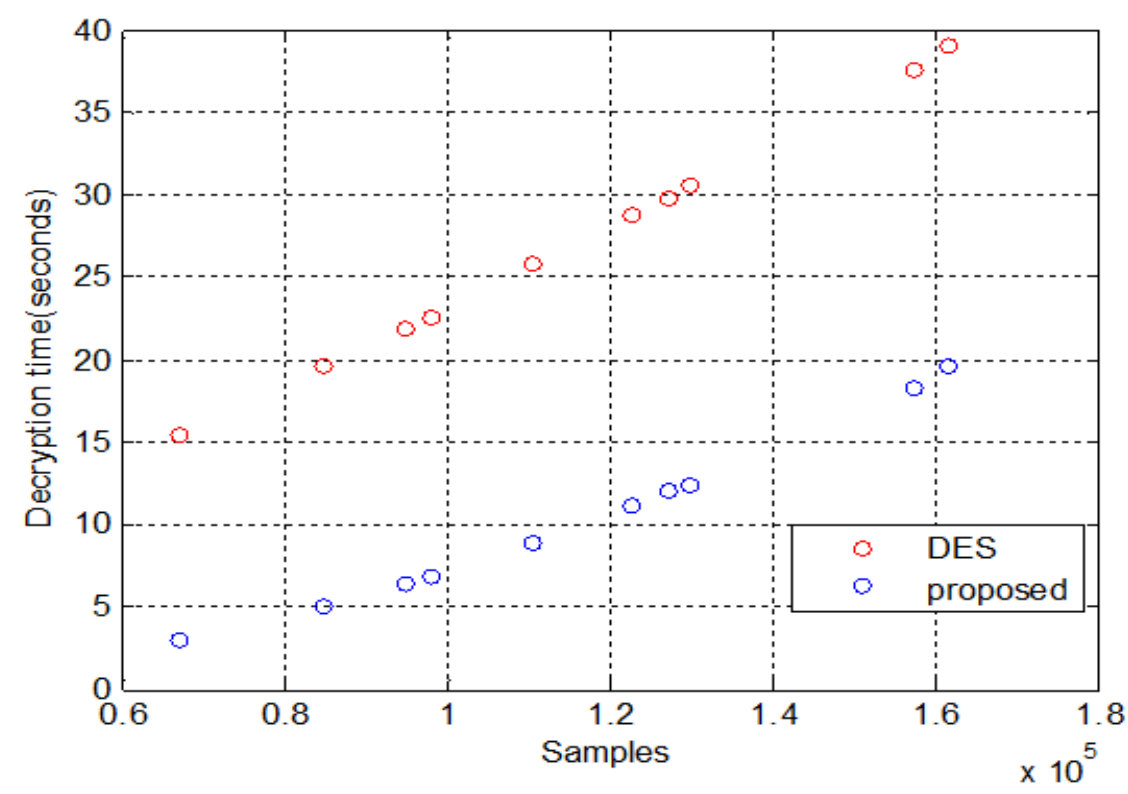

Figure 7: Decryption times comparisons

\section{CONCLUSION}

A simple and easy to implement method of speech signal cryptography was introduced and tested. The method is flexible giving us a chance to use various windows and various private keys with various lengths. The proposed is highly secure because it very difficult to hack the private key. The proposed method was compared with DES method and it was shown that the proposed method is more efficient by decreasing the encryption and decryption times, thus increasing the method throughput. The proposed method was tested for quality and it gave good value for the quality parameter PSNR in both phases: encryption phase and decryption phase.

\section{FUNDING STATEMENT}

This work was supported by the Taif University Researchers Supporting Project Number (TURSP-2020/345), Taif University, Taif, Saudi Arabia.

\section{ACKNOWLEDGMENT}

This work was supported by the Research Groups Program Funded by Deanship of Scientific Research, Taif University, Ministry of Education. Saudi Arabia, under Grant (TURSP-2020/345).

\section{REFERENCES}

1. K. Matrouk, A. Al-Hasanat, H. Alasha'ary, Z. Al-Qadi, H. Al-Shalabi, Speech fingerprint to identify isolated word person, World Applied Sciences Journal,2014, 31 (10), 17671771.

2. Aws Al-Qaisi, Saleh A Khawatreh, Ahmad A Sharadqah, Ziad A Alqadi, Wave file features extraction using reduced LBP, International Journal of Electrical and Computer Engineering, 2018, 8, 5,2785. 
3. R.J.Rasras, B. Zahran, M.R.A. Sara, Z. AlQadi, Developing digital signal clustering method using local binary pattern histogram, International Journal of Electrical and Computer Engineering, 2021, 11,1, 872-878.

4. Dr. Mohammad S. Khrisat, Prof. Ziad Alqadi, Simple, Qualities, Efficient and Secure Method to Encrypt Voice Signal, International Journal of Computer Applications, 2021,183, 7, 25-29, 2021.

5. Prof. Ziad Alqadi, A Simple Method To Encrypt-Decrypt Speech Signal, IJETRM, 2021, 5, 2, 44-52, 9-2785.

6. Jihad Nader Ismail Shayeb, Ziad Alqadi, Analysis of digital voice features extraction methods, International Journal of Educational Research and Development, 2019,1, 4, 49-55.

7. Amjad Hindi, Majed Omar Dwairi, Ziad Alqadi, Analysis of Digital Signals using Wavelet Packet Tree, IJCSMC, 2020, 9, 2, 96-103.

8. Yousf Eltous Ziad A. AlQadi, Ghazi M. Qaryouti, Mohammad Abuzalata, Analysis Of Digital Signal Features Extraction Based On Kmeans Clustering, International Journal of Engineering Technology Research \& Management, 2020, 4,1, 66-75.

9. Prof. Ziad Alqadi, Efficient And Highly Secure Method Of Message Encryption, IJETRM,2021, 5, 2, 58-64.

10. Holwa Fayeq Taha, Prof. Ziad Alqadi, Speech Signal Cryptography Using WPT, International Journal of Engineering Technologies and Management Research,2020, 7, 7,71-79.

11. Saleh Khawatreh, Belal Ayyoub, Ashraf Abu-Ein, Ziad Alqadi, A Novel Methodology to Extract Voice Signal Features, International Journal of Computer Applications, 2018,179 -9 .

12. Ayman Al-Rawashdeh, Ziad Al-Qadi, Using wave equation to extract digital signal features, Engineering, Technology \& Applied Science Research, 2018, 8, 4,1356-1359.

13. Majed O Al-Dwairi, A Hendi, Z AlQadi, An efficient and highly secure technique to encrypt-decrypt color images, Engineering, Technology \& Applied Science Research, 2019, vol. 9, issue 3, pp. 4165-4168, 2019.

14. Amjad Y Hendi, Majed O Dwairi, Ziad A Al-Qadi, Mohamed S Soliman, A novel simple and highly secure method for data encryption-decryption, International Journal of Communication Networks and Information Security,2019,11, 1, 232-238.

15. Mutaz Rasmi Abu Sara Rashad J. Rasras, Ziad A. AlQadi, A Methodology Based on Steganography and Cryptography to Protect Highly Secure Messages, Engineering, Technology \& Applied Science Research, 2019, 9,1, 3681-3684.

16. A Waheeb, Ziad AlQadi, Gray image reconstruction, Eur. J. Sci. Res, 2009, 17, 167-173.

17. Jihad Nader, Ziad A. A. Alqadi, Bilal Zahran, Analysis of Color Image Filtering Methods, International Journal of Computer Applications, 2017,174, 8, 12-17.

18. Jamil Al-Azzeh, Ziad Alqadi, Qazem Jaber, A Simple, Accurate and Highly Secure Method to Encrypt-Decrypt Digital Images, JOIV: International Journal on Informatics Visualization, 2019, 3, 3,262-265. 
19. Ziad Alqadi, Bilal Zahran, Jihad Nader, Estimation and Tuning of FIR Lowpass Digital Filter Parameters, International Journal of Advanced Research in Computer Science and Software Engineering, 2017, 7, 2,18-23.

20. Ziad Alqadi, Bilal Zahran, Qazem Jaber, Belal Ayyoub, Jamil Al-Azzeh, Ahmad Sharadqh, Proposed Implementation Method to Improve LSB Efficiency, International Journal of Computer Science and Mobile Computing, 2019, 8, 3, 306-319.

21. Mohammad S. Khrisat, Rushdi. S. Abu Zneit, Hatim Ghazi Zaini, Ziad A. Alqadi, Analysis Methods Used to Extract Fingerprints Features, Traitement du Signal,2021, 38, 3, 711-717.

22. Dr. Mohammad S. Khrisat Prof. Ziad Alqadi, Color Images Classifier Optimization, International Journal of Engineering Technology Research \& Management,2021,5, 5, 614 .

23. Ziad alqadi, Analysis of stream cipher security algorithm, Journal of Information and Computing Science, 2007, 4, 2, 288-298.

24. Belal Zahran Rashad J Rasras, Ziad Alqadi, Mutaz Rasmi Abu Sara, B Zahran, Developing new Multilevel security algorithm for data encryption-decryption (MLS_ED), International Journal of Advanced Trends in Computer Science and Engineering, 2019,8, 6, 3228-3235.

* Corresponding Author:Prof. Ziad A. Alqadi,

Albalqa applied university, Jordan

Online date of publication: 26.10.2021 